\title{
Static Zero-Power-Consumption Coplanar Waveguide Embedded DC-to-RF Metal-Contact MEMS Switches in Two-Port and Three-Port Configuration
}

\author{
Mikael Sterner, Niclas Roxhed, Member, IEEE, Göran Stemme, Fellow, IEEE, and \\ Joachim Oberhammer, Member, IEEE
}

\begin{abstract}
This paper reports on novel electrostatically actuated dc-to-RF metal-contact microelectromechanical systems (MEMS) switches, featuring a minimum transmission line discontinuity since the whole switch mechanism is completely embedded inside the signal line of a low-loss 3-D micromachined coplanar waveguide. Furthermore, the switches are based on a multistable interlocking mechanism resulting in static zero-power consumption, i.e., both the onstate and the offstate are maintained without applying external actuation energy. Additionally, the switches provide with active opening capability, potentially improving the switch reliability, and enabling the usage of soft low-resistivity contact materials. Both two-port single-pole-single-throw (SPST) switches featuring mechanical bistability and three-port singlepole-double-throw (SPDT) T-junction switches with four mechanically stable states are presented. The switches, together with the transmission lines, are fabricated in a single photolithography process. The loss created by the discontinuity of the switch mechanism alone is $0.08 \mathrm{~dB}$ at $20 \mathrm{GHz}$. Including a $500 \mu \mathrm{m}$ long transmission line with less than $0.4 \mathrm{~dB} / \mathrm{mm}$ loss up to $20 \mathrm{GHz}$, the total insertion loss of the two-port devices is 0.15 and $0.3 \mathrm{~dB}$ at 2 and $20 \mathrm{GHz}$, and the isolation is 45 and $25 \mathrm{~dB}$ at 2 and $20 \mathrm{GHz}$. The three-port switches, including their T-junction transmission line, have an insertion loss of 0.31 and $0.68 \mathrm{~dB}$, and an isolation of 43 and $22 \mathrm{~dB}$, at 1 and $10 \mathrm{GHz}$, respectively. Actuation voltages are 23-39 $\mathrm{V}$ for the two-port switches and 39-89 $\mathrm{V}$ for the threeport switches. The microwave propagation in the micromachined transmission line and the influence of the different switch designs were analyzed by finite-element method (FEM) simulations of electromagnetic energy and volume current distributions, proving the design advantages of the proposed concept.
\end{abstract}

Index Terms-Electrostatic actuator, RF microelectromechanical systems (MEMS), switch.

\section{INTRODUCTION}

M ICROELECTROMECHANICAL systems (MEMS) metal-contact switches are microrelays fabricated by semiconductor manufacturing techniques. Due to their submillimeter size, achieved by wafer-scale integration using standard high-volume semiconductor manufacturing technology,

Manuscript received June 11, 2009; revised February 19, 2010; accepted March 30, 2010. Date of publication May 24, 2010; date of current version June 23, 2010. This paper was reviewed by Editor C. Nguyen.

The authors are with the Microsystem Technology Lab, Royal Institute of Technology (KTH), Stockholm 10044, Sweden (e-mail: msterner@kth.se).

Color versions of one or more of the figures in this paper are available online at http://ieeexplore.ieee.org.

Digital Object Identifier 10.1109/TED.2010.2048239 and due to their ohmic switching behavior, they offer superior signal handling performance as compared to solid-state relays or miniaturized electromagnetic relays: low insertion loss, high isolation, excellent signal linearity, and a very large bandwidth with these good signal properties. MEMS metal-contact switches, in contrast to capacitive switches, can handle signals from dc up to many tens of $\mathrm{GHz}[1]-[3]$.

Electrostatic actuation is the preferred operation principle for MEMS switches because of its large actuation forces, sufficient displacement for RF signal switching, very low power consumption (including the driving circuitry), good reliability, and suitability for high-volume wafer-scale manufacturing using standard semiconductor processes and materials [4]. Most switch designs are based on elements moving perpendicular to the wafer surface, requiring complicated multilayer surface micromachining techniques [5]-[7]. Laterally in-wafer-plane moving actuators, on the other hand, allow for uncomplicated bulk micromachining fabrication techniques with a drastically reduced number of process steps, and have already been employed for inline microswitches [8], [9]. However, laterallymoving designs previously reported in the literature, including previous work by the authors [10], are not suitable for switching RF signals since the rigid electrode of the actuator, typically even connected to the ground, must be placed in close vicinity to the moving electrode carrying the RF signal, which creates a large capacitive load resulting in a low characteristic impedance and thus imposing a heavy discontinuity to the transmission line.

The lifetime of MEMS metal-contact switches is typically limited by contact stiction. MEMS switch designs are very vulnerable to this failure mode because they usually rely on a passive mechanical spring for opening the switch contacts. Push-pull mechanisms, on the other hand, are based on active electrostatic actuation both for closing and for opening the switch [11], [12], which has the drawback of a more complicated fabrication and operation. In a previous work, the authors have shown switch designs employing a passive closing force, still sufficient to create a low-resistance contact with softmetal contact materials, and a strong active opening force, for overcoming stiction [10], [13].

Most switches are mechanically monostable, i.e., they maintain only one position, typically the offstate, without 
applying external energy. For the other state, even an electrostatically actuated switch has a nonnegligible power consumption because of its driving circuit. Mechanically multistable switches, however, have multiple mechanically stable states. For such switch mechanisms, external energy is only needed for the transition between the mechanically stable states and they are therefore classified as static zero-power switches [14].

Micromachining techniques have also been employed to fabricate very low-loss transmission lines by reducing dielectric losses in materials exposed to the electromagnetic field of the line. Examples include etching away parts of the lossy substrate close to the line [15]-[17]; creating hollow waveguides by bonding substrates with etched grooves [18], [19]; suspending elements of the transmission line above the lossy substrate by etching away sacrificial layers below them [20]-[23]; and using 3-D polymer or metal-printing techniques to achieve air-filled transmission lines [24]-[26] or coaxial waveguides filled with low-loss polymers [27]. Reduction of ohmic losses by increasing the metal thickness of coplanar waveguides has been shown using synchrotron radiation lithography [28].

This paper reports an electrostatically actuated laterally moving metal-contact RF MEMS switch which, in a unique way, combines the following: 1) low-intrusive RF design by embedding the complete switch mechanism inside the signal line of a coplanar waveguide; 2) integration into a low-loss 3-D micromachined coplanar waveguide; 3) a robust static zero-power consumption mechanically multistable actuator with active opening capability; and 4) uncomplicated fabrication by a single photolithography step.

A bistable mechanism has been implemented for a singlepole-single-throw (SPST) two-port switch. Furthermore, a single-pole-double-throw (SPDT) three-port embodiment has been implemented using a single quad-stable mechanism embedded inside a T-junction of a transmission line, in contrast to all previously presented RF MEMS SPDT switch designs, where either two separate SPST switches are used [29]-[31] or a single mechanism is used with two separate switch contacts [32], all typically positioned at $\lambda / 4$ distance from the junction.

In comparison to an early paper published by the authors on laterally moving interlocking cantilever switches [10], [14], which were fundamentally unsuitable for switching RF signals, this paper presents a concept which uniquely separates the function of the small gap between the actuator electrodes from the function of the large signal-to-ground gap of the transmission line, enabling it for switching microwave signals. The basic concept of a transmission-line embedded SPST switch mechanism was introduced by the authors at a conference presentation [33], and the SPDT embodiment was shown at a follow-up meeting [34]. The present paper presents a more complete study on these types of multistable switch mechanisms, enhancing the previously presented conference papers by more device embodiments, an analysis of the wave propagation in the micromachined transmission lines, T-junctions and switch mechanisms, and more characterization data on the MEMS actuation mechanism.

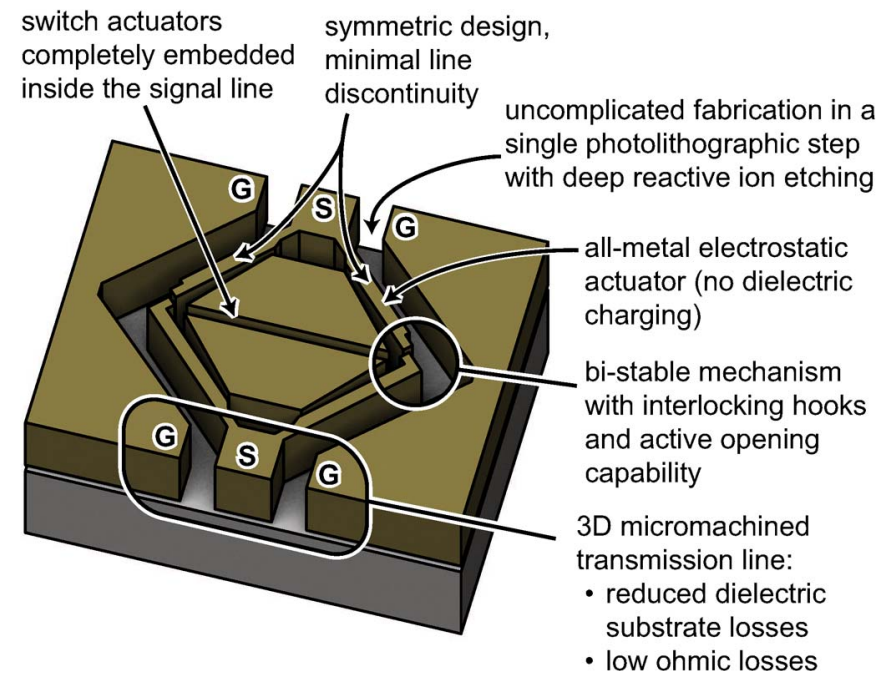

Fig. 1. Conceptual illustration of the presented static zero-powerconsumption metal-contact RF MEMS switch integrated in a 3-D micromachined coplanar waveguide.

\section{Switch Concept And Design FeAtures}

The electrostatically actuated metal-contact RF MEMS switch concept reported in this paper is illustrated in Fig. 1, giving an overview of the main features. The concept combines the following special features in a very unique way:

1) Mechanical multistability: The present switch designs are fully mechanically stable in both the onstate and in the offstate, i.e., the states are maintained without applying any external actuation energy. Thus, the switch is truly static zero-power-consuming, with external voltage only needed for the transition between its states. The mechanical stability of every state is achieved by a mechanism with two perpendicularly arranged cantilevers with interlocking hooks. The actuation sequence for interlocking and for unlocking the cantilevers in the transition between the on and the offstate is shown in Fig. 2 for a twoport bistable device. The three-port devices have four mechanically stable states.

2) Active opening capability: In the presented concept, the transition from the onstate to the offstate is done by actively separating the contacts by electrostatic actuation, in contrast to most MEMS switches which are passively opened by a very limited restoring spring force, making them susceptible to the major MEMS switch failure of permanent contact stiction. Especially soft materials, favored in designing MEMS switches because of their low resistance, develop large adhesion forces between the metal contacts. For the present switch concept, the contact force is created passively by the deflected interlocked hooks, and the opening force is created actively by applying the actuation voltage for disconnecting the cantilevers. This results in a large opening force controllable by the external actuation voltage, potentially improving the switch reliability as compared to conventional MEMS switch concepts, which was investigated previously by the authors for interlocking-hook mechanisms, showing 


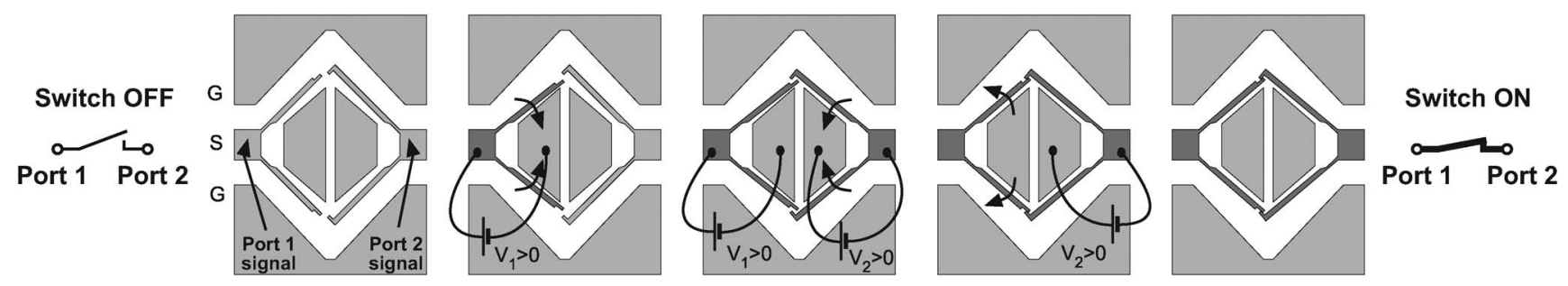

Fig. 2. Actuation sequence for closing and opening the presented mechanically bistable coplanar-waveguide integrated switch by interlocking and unlocking cantilevers with hooks. Since the on and offstates are mechanically stable, the two control potentials are only applied during the transition between the states.

that active opening forces potentially up to $2 \mathrm{mN}$ could be achieved for this type of switch design [10].

3) Very low intrusive RF design: The switch actuation mechanism is completely placed inside the signal line of the coplanar waveguide transmission line. This is possible since the currents in a coplanar waveguide are mainly confined to the edges of the conductors, thus leaving the inside of the signal line field-free. Two switch mechanisms are placed symmetrically on each side of the signal line to maintain a balanced wave propagation mode in the two signal-to-ground gaps. The line continuity on the sidewalls of the signal line is ensured by the switch cantilevers being placed on the outside of the switch mechanism.

4) Single photolithography step fabrication of the switches together with the 3-D transmission lines using bulk micromachining deep reactive ion etching, comprising very simple fabrication by a minimum number of standard fabrication steps. For a more complete switch design, biasing lines as used in conventional capacitive switches, e.g., as well as packaging and back-end processing, will require additional process steps.

5) Monocrystalline silicon used as structural material for all moving parts, providing best possible mechanical reliability, substantially better than deposited amorphous $\mathrm{SiN}$ or $\mathrm{SiO}_{2}$ or electroplated metal structures as used in conventional switch designs.

6) Temperature compensation: The symmetrical Au-Si-Au metallization of the silicon cantilevers, as shown in Fig. 6, eliminates susceptibility to changes in the operation temperature.

7) All-metal switch actuators with stoppers for avoiding short-circuit between the switch elements, providing robust actuation and stable actuation voltages. This is in contrast to conventional electrostatic MEMS switches, where charges are trapped in the dielectric layers used for isolation, due to the high field strength in the electrostatic actuators, leading to unstable and nonreproducible actuation voltages or even rendering the switches inoperable.

8) 3-D micromachined coplanar waveguide providing low dielectric substrate losses and low ohmic losses. (See next section.)

As compared to these advantages, the following peculiarities of this novel concept must be considered:

1) Actuation time: The current design requires a more complex actuation control sequence than conventional electrostatic switches, due to the closing and opening of the interlocking switch elements in separate phases. Thus it requires more complex driving circuitry and the actuation time is $4-5$ times longer, but still, by far, faster than electrothermal actuator-based designs.

2) Mechanical design: The interlocking mechanism requires a more elaborate design phase as compared to conventional single-cantilever switches.

3) Fabrication: The concept requires silicon-on-insulator (SOI) wafers which are still more expensive than standard wafers, though these costs are compensated for by the much simpler fabrication procedure than for conventional surface-micromachined switches.

4) Contact force: Even though the active opening force concept is capable of creating large opening forces [10] making this concept very suitable for soft-contact materials, the contact force is created passively which makes it less suitable for hard-contact materials [35].

\section{Design of 3-D Micromachined Vertically EXTENDED COPLANAR WAVEGUIDES}

Conventional 2-D coplanar waveguides consist of thin-film conductive metal lines directly deposited on the substrate. Thus, the substrate is penetrated by a major part of the electric field lines, as shown in Fig. 3(a), which causes dielectric losses. Micromachining allows to fabricate 3-D transmission lines, i.e., extending the planar metal lines into the third dimension, where the major part of the field lines is confined outside the substrate, as shown in Fig. 3(b), reducing substrate losses. Also, the ohmic losses are reduced since the current has a much larger volume available in the sidewalls, due to the skin-effect limiting the current mainly in the lateral dimension.

The 3-D micromachined coplanar waveguides utilized for the switches in this paper are created by depositing a conductive metal layer on the sidewalls of deep trenches in the silicon device layer of a SOI wafer [Fig. 3(b)]. The thickness of the device layer determines the height of the transmission line, and the thickness of the buried-oxide (BOX) insulation layer limits the capacitive coupling between the lower end of the metal conductors and the handle wafer substrate. A conventional 2-D coplanar waveguide would correspond, in this parametrization, to having zero-thickness device and BOX layers, i.e., with the metal lines directly on the SOI handle substrate [Fig. 3(a)]. With this in mind, full-field electromagnetic simulations of the transmission line geometry with different layer thicknesses were run to compare the properties of the 3-D coplanar waveguide to the 2-D transmission line. 


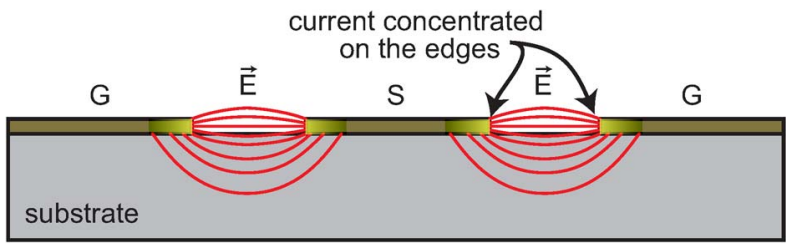

(a)

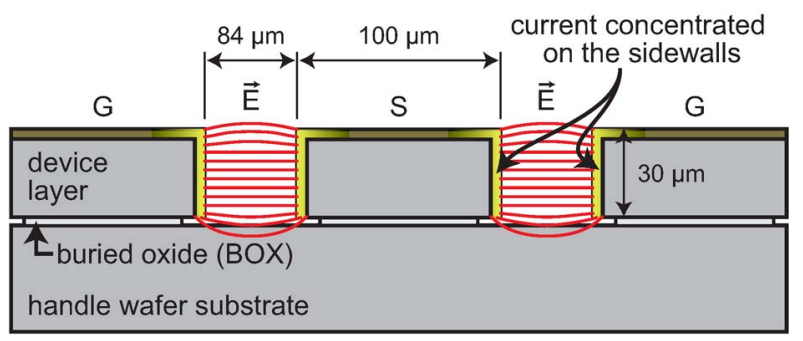

(b)

Fig. 3. A comparison between the cross-sections of conventional 2-D coplanar waveguides and the 3-D micromachined coplanar waveguide of this paper. (a) A conventional coplanar transmission line, consisting of thin conductive metal lines on the substrate. Most of the electric field lines penetrate the substrate causing losses. (b) The 3-D micromachined coplanar waveguide used in this paper. Most of the electric field is confined to the air between the lines dramatically reducing propagation losses in the substrate.

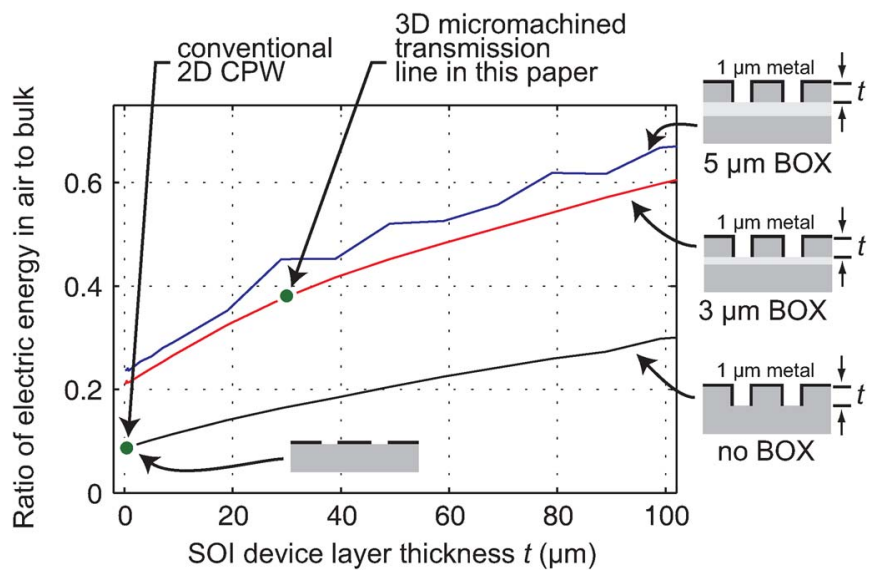

Fig. 4. Simulation ratio between the electric energy $\left(\int \varepsilon E^{2}\right)$ contained outside the substrate to the energy in the lossy substrate of the transmission line cross-section, extracted from a full-field HFSS simulation at $10 \mathrm{GHz}$.

The dielectric substrate losses in the transmission line are related to the concentration of the electric field energy in the substrate. Thus, the simulated total electric field energy in the substrate, as compared to the total energy outside the substrate, is a measure for the efficiency of the transmission line. In Fig. 4, the simulated ratio of electric field energy $\left(\int \varepsilon E^{2}\right)$ in air to bulk is given as a function of the SOI device layer thickness for three different configurations, namely, no BOX layer, $3 \mu \mathrm{m} \mathrm{BOX}$ layer and $5 \mu \mathrm{m} \mathrm{BOX}$ layer, for a metal layer thickness of $1 \mu \mathrm{m}$ on top and on the sidewalls. The data points representing a conventional 2-D coplanar waveguide and the 3-D micromachined coplanar waveguide employed in this paper are highlighted. The 3-D transmission line has four times higher ratio as compared to the 2-D conventional line. Increasing the device layer thickness further increases the ratio. The BOX layer drastically reduces the substrate coupling, as compared to the configuration without the BOX layer, but increasing the

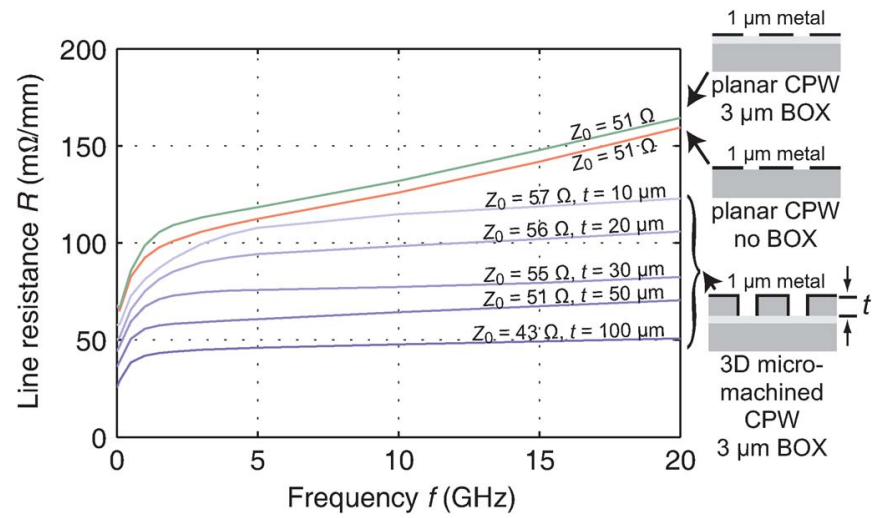

Fig. 5. Simulation frequency dependency of the line resistance of different transmission line configurations extracted from a full-field HFSS simulation. The characteristic impedance $Z_{0}$ is given for $10 \mathrm{GHz}$.

BOX layer thickness from $3 \mu \mathrm{m}$ to $5 \mu \mathrm{m}$ only shows a marginal improvement.

The ohmic losses of the transmission line are proportional to its frequency dependent line resistance. In Fig. 5, the simulated frequency-dependent line resistance is shown for the 3-D micromachined coplanar waveguide in configurations of 10- to $100-\mu \mathrm{m}$-thick device layers, compared to the conventional 2-D coplanar waveguide in the two configurations with and without $B O X$ layer. The line resistance is extracted from the integrated volume loss density $P$ as $R=2 P / I^{2}$, where $I$ is the integrated volume current through a cross-section of the metal line [36], both obtained by FEM simulations with Ansoft HFSS. The metal-covered sidewalls of the 3-D micromachined transmission line clearly give a lower resistance than the conventional 2-D coplanar waveguides, and the resistance decreases when the device layer thickness is increased, as expected from the increase in the cross-sectional area of the region at the edges of the metal lines, where the current is concentrated (Fig. 3).

\section{SOI RF MEMS FABRICATION TECHNOLOGY}

Fabricating inplane moving RF MEMS devices in SOI device layers using bulk micromachining allows for advanced designs with complex lateral features defining both the circuit and actuator elements but with uncomplicated fabrication.

The process flow used for the fabrication is shown in Fig. 6. Structures geometrically connected in the 2-D layout are also electrically connected by a full-wafer metallization step, whereas geometrically unconnected structures are electrically isolated by the buried oxide. The whole geometry is defined by a single photolithography step. Deep-reactive-ion etching (DRIE) with a $\mathrm{SiO}_{2}$ hard mask is used to structure the 30- $\mu \mathrm{m}$-thick device layer of a high-resistivity SOI wafer [Fig. 6(a) and (b)]. Hydrofluoric acid is used to underetch the buried oxide layer of the SOI wafer which releases the moving cantilevers [Fig. 6(c)]. The underetching of the wet etching step guarantees that the device layer metallization is electrically disconnected from the substrate metallization during the subsequent metal deposition process, where a 800-nm-thick layer of gold on a 100-nm titanium adhesion layer is deposited using sputter-coating [Fig. 6(d)]. The metal coating on the handle wafer in the coplanar waveguide slots is removed by maskless 


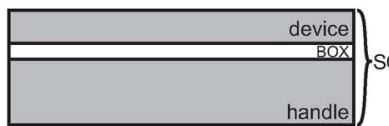

(a)

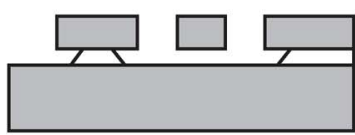

(c)

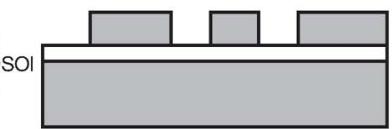

(b)

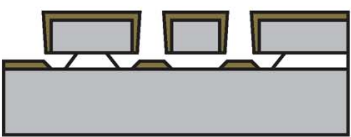

(d)
S

$\mathrm{SiO}_{2} \square \mathrm{Au}$

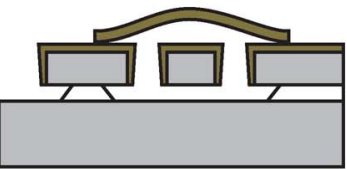

(e)

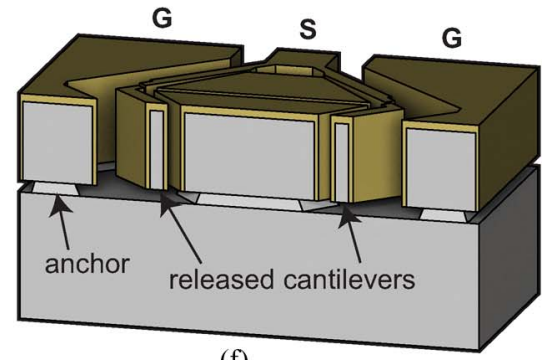

(f)

Fig. 6. Basic process flow of the switch fabrication: (a) SOI wafer; (b) patterning and etching the device layer; (c) release-etching of the moving structures by wet etching of the SOI-BOX layer; (d) sputter-coating with gold; (e) final devices with the metal coating on the substrate removed and the ground lines connected by wire-bonded air bridges; (f) cross-sectional 3-D rendering of the transmission line embedded switch, showing the moving cantilever structures in the signal line.

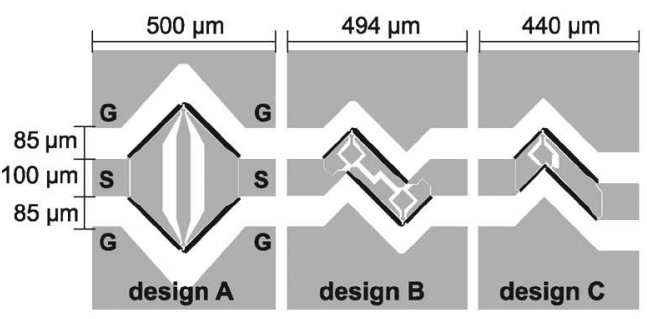

(a)

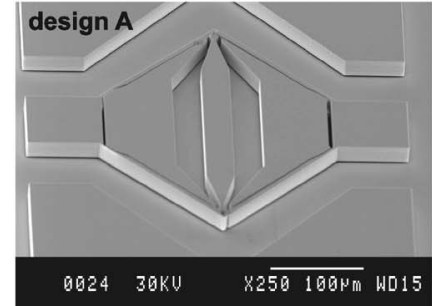

$002430 \mathrm{KV}$

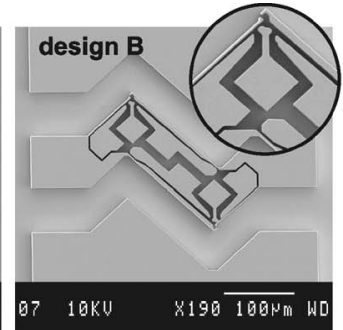

(b)

Fig. 7. Design variants of the two-port SPST devices: (a) Drawings of the three basic designs of the coplanar waveguide embedded switch concept. The interlocking cantilevers for mechanical bistability are emphasized in the drawings. (b) SEM pictures showing fabricated devices of the three designs.

localized electrochemically assisted etching, a fabrication technique previously introduced for this purpose by the authors [37]. For the three-port devices, the opposing ground lines in the corners of the T-junction are electrically connected by wirebonded air bridges to balance the desired coplanar waveguide mode [Fig. 6(e)]. A cross-sectional 3-D rendering is presented in Fig. 6(f), showing the released cantilevers after fabrication.

\section{Two-PorT And THREE-PORT EMBODIMENTS}

\section{A. Two-Port Device: Mechanically Bistable SPST Switch}

The concept of the mechanically bistable SPST switch embedded in a coplanar waveguide has been implemented in three different design variants, which are shown in Fig. 7 together with scanning electron microscopy (SEM) pictures of fabricated devices. The difference between the designs is in how the switch elements are embedded into the signal line. Design A uses four $200-\mu \mathrm{m}$-long cantilevers arranged symmetrically, whereas designs B and $\mathrm{C}$ each use two 100- $\mu \mathrm{m}$ and two $200-\mu \mathrm{m}$ cantilevers. All interlocking cantilevers are arranged at $90^{\circ}$ angle to each other. The arrangement in design A preserves the orientation of the of the signal line, but varies the signal line width, which must be compensated by adjusting the signalto-ground distance for constant characteristic impedance. In designs $\mathrm{B}$ and $\mathrm{C}$, the cross-sectional width of the signal line is kept constant but the centerline is locally offset from the general transmission line direction. Design $\mathrm{C}$ has one corner less than design $\mathrm{B}$, at the expense of a remaining lateral offset between the incoming and outgoing signal line. The ends of the cantilevers are endowed with interlocking hooks to provide mechanical bistability (as shown in Fig. 2). SEM pictures
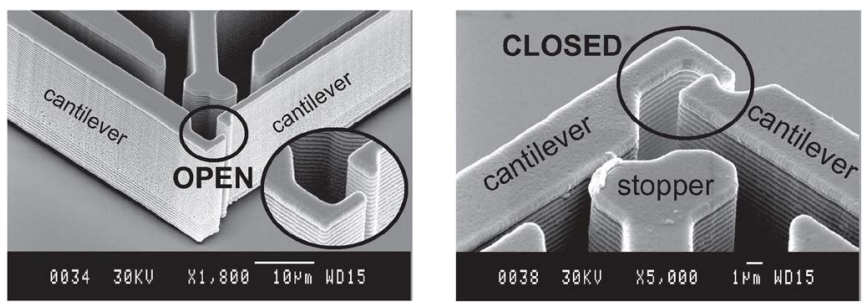

Fig. 8. Closeup view SEM pictures of the hook mechanism: (a) unlocked state (open switch), (b) locked state (closed switch).

showing a closeup view of the hook mechanism is shown in Fig. 8. The isolation in the offstate is given by the distance between the cantilever hooks of $2.5 \mu \mathrm{m}$ for all three design variants. The relative trajectories of the cantilevers are designed to give just enough clearance for the movement and interlocking of the hooks. Additionally, two of the cantilevers in each design are designed to provide a large deflection and the other two for providing a large contact force. This allows a large separation in the offstate without sacrificing the contact performance in the onstate. The large-deflection cantilevers have a total movement of $4.5 \mu \mathrm{m}$ and a spring constant of $2 \mathrm{~N} / \mathrm{m}$, as compared to $2.5 \mu \mathrm{m}$ and $11 \mathrm{~N} / \mathrm{m}$, respectively for the large-contact-force cantilevers, resulting in a total contact force of approximately $30 \mu \mathrm{N}$, sufficient for sputtered soft-gold designs [10].

\section{B. Three-Port Device: Quad-Stable SPDT Switch}

A quad-stable switch mechanism has been embedded in a coplanar waveguide T-junction, for a three-port SPDT switch. Two different designs of the SPDT embodiment were implemented and are shown in Fig. 9. Both designs are symmetric across the center line, featuring cantilevers along the outside 

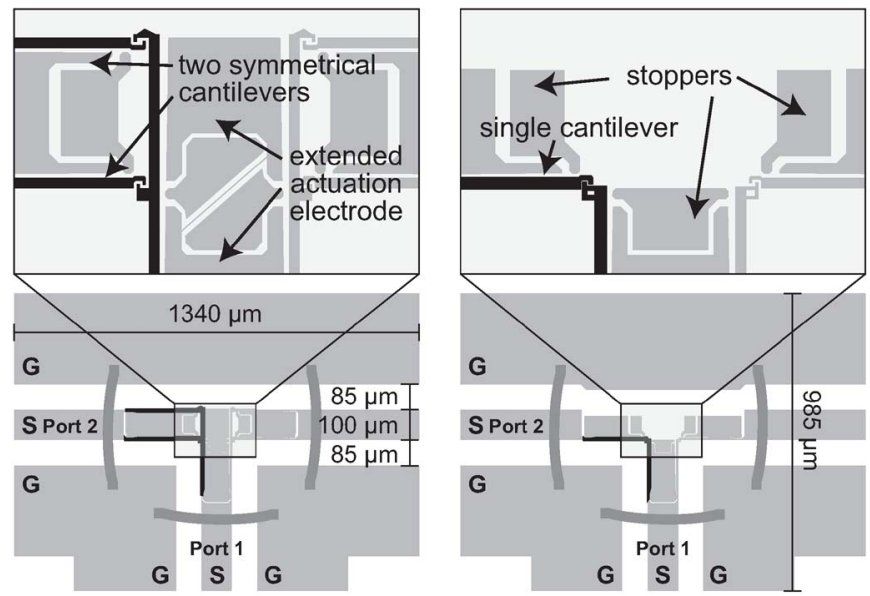

design $D$
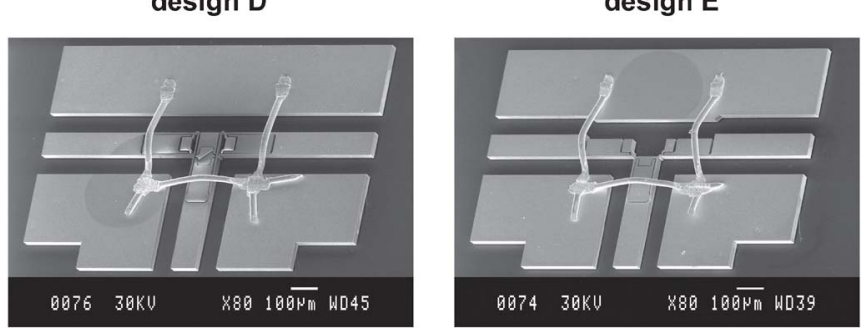

Fig. 9. Drawings of the two three-port SPDT switch designs and SEM pictures of fabricated devices. The interlocking cantilevers for connecting the signal line between port 1 and port 2 are emphasized.

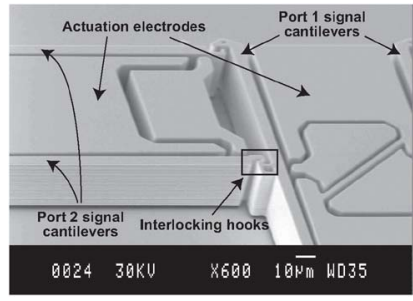

(a)

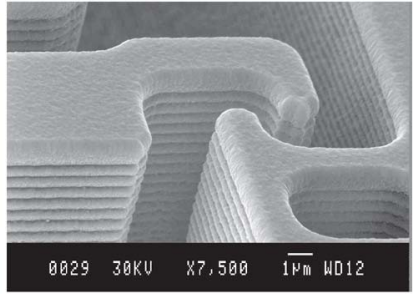

(b)
Fig. 10. Close-up SEM pictures of a fabricated T-junction switch. (a) The interlocking mechanism on one side of the SPDT witch. (b) Closeup view of the hook configuration in the open switch state.

of the signal lines. The cantilevers along the signal lines of the output ports (port 2 and port 3 ) interlock with the cantilevers along the signal line of the input port (port 1). In design D, there are two hooks on each cantilever of the input signal line and two symmetrically arranged cantilevers along the signal lines of the outputs. Design E has only one hook on the cantilevers of the input and a single asymmetric cantilever at the outputs. In both designs, the cantilevers are $300 \mu \mathrm{m}$ long for the input and $200 \mu \mathrm{m}$ for the outputs. The ground lines of the coplanar waveguides in T-junction configuration are connected by three air bridges located close to the switch mechanism to balance the symmetrical coplanar mode. Fig. 10 shows SEM closeup pictures of a fabricated SPDT switch and features of the interlocking switch mechanism for the symmetrical cantilever configuration.

The mechanically quad-stable mechanism of the SPDT embodiment is illustrated in Fig. 11, showing the transitions between the four stable states, namely, switch open, closed between port 1 and port 2, closed between port 1 and port 3 ,

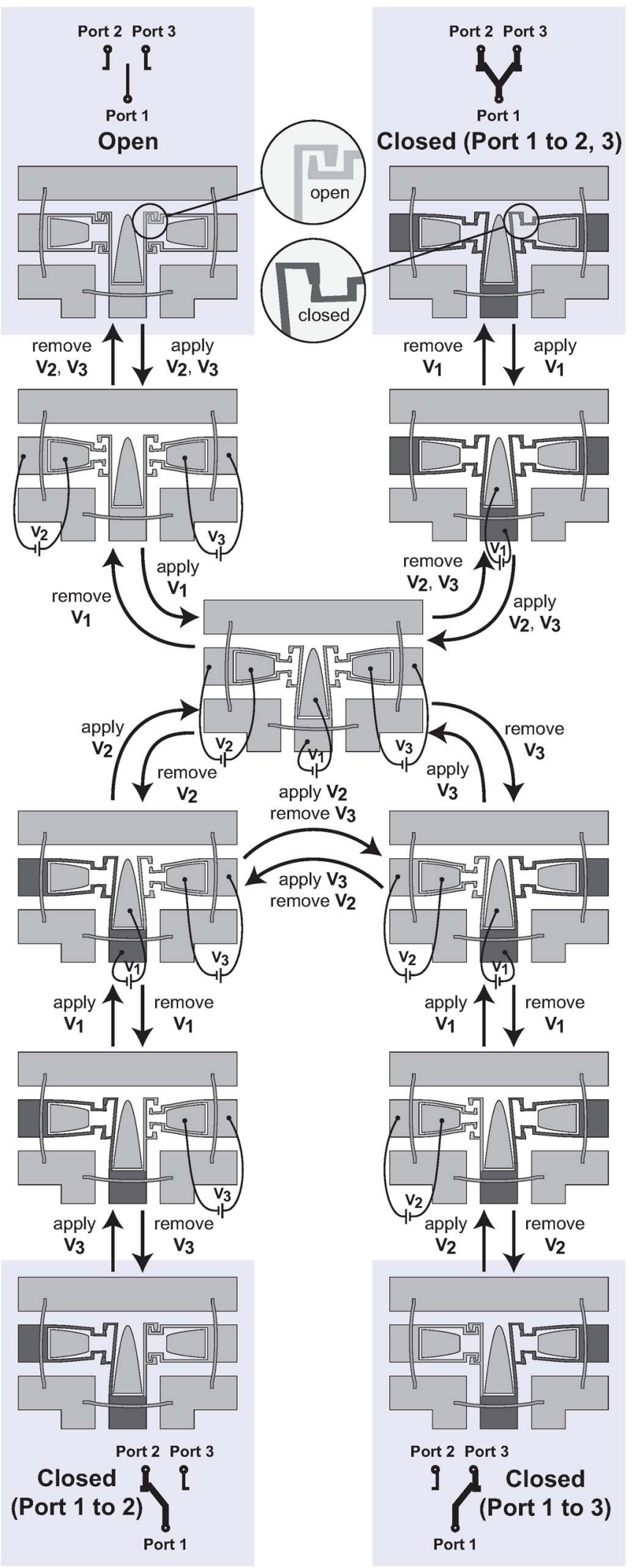

Fig. 11. Illustration of the four mechanically stable states of the threeport switch embodiment $\mathrm{D}$, including the transitional states when changing configuration between these states. The annotations at the arrows indicate the change in the actuation voltage configuration necessary for the transition between the different states. 

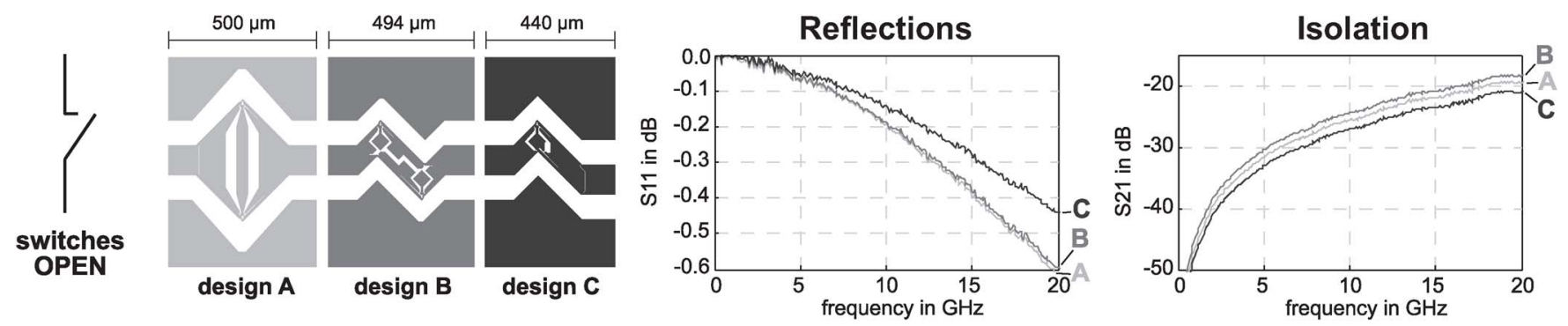

Fig. 12. RF evaluation of the three SPST switch designs A, B, and C in the open state.
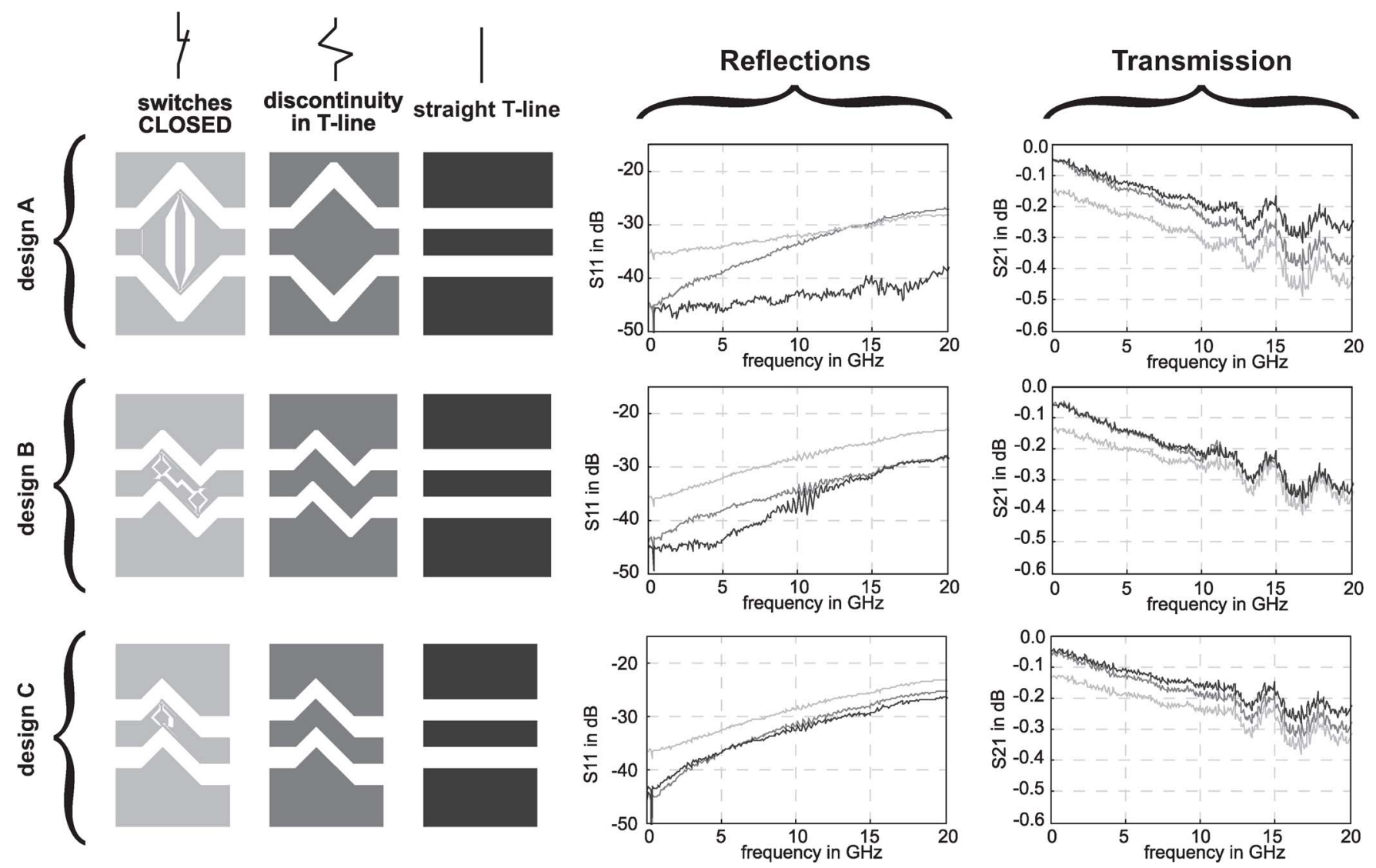

Fig. 13. RF evaluation of the three switch designs A, B, and C in the closed state, and in comparison to the transmission line discontinuity of the switch geometry alone and to straight transmission line pieces of the same length.

and closed between port 1 and both output ports ( 2 and 3 ). The actuation sequence allows for transitioning between any of the stable states in at most five steps. Only three control potentials are required, since the cantilevers along the signal line at port 1 share a common actuator electrode. The possibility of connecting port 1 to both port 2 and port 3 simultaneously enables the use of the device as a switchable power splitter, in which case the transmission lines at port 2 and 3 should be designed for a characteristic impedance twice that of the transmission line at port 1 .

\section{EVALUATION}

\section{A. Performance of the Two-Port Switches}

The RF performance of the devices has been evaluated with an Agilent E8361A PNA Network Analyzer, calibrated with a GGB Industries CS-5 calibration substrate. Fig. 12 shows the isolation S21 and the reflections S11 of the three switch designs in the open state. Furthermore, Fig. 13 compares the RF performance of the closed switches to transmission line geometries representing the switch discontinuities and to straight transmission line pieces of equal length. The total insertion loss of the best design (C) including its transmission line was measured to less than 0.15 and $0.35 \mathrm{~dB}$ at 2 and $10 \mathrm{GHz}$, respectively. The isolation for the same design was determined to 45 and $25 \mathrm{~dB}$ at 2 and $10 \mathrm{GHz}$, respectively.

The 3-D micromachined transmission line alone was found to have a loss of less than $0.4 \mathrm{~dB} / \mathrm{mm}$ up to $10 \mathrm{GHz}$.

Beside the low reflections in the onstate, the minimum RF intrusiveness of the novel switch mechanism concept is demonstrated by the low switch insertion loss created by the mechanism alone (i.e., corrected by subtracting the transmission line losses) amounting to less than $0.1 \mathrm{~dB}$ up to $20 \mathrm{GHz}$ for the designs B and C, as shown in Fig. 14. 


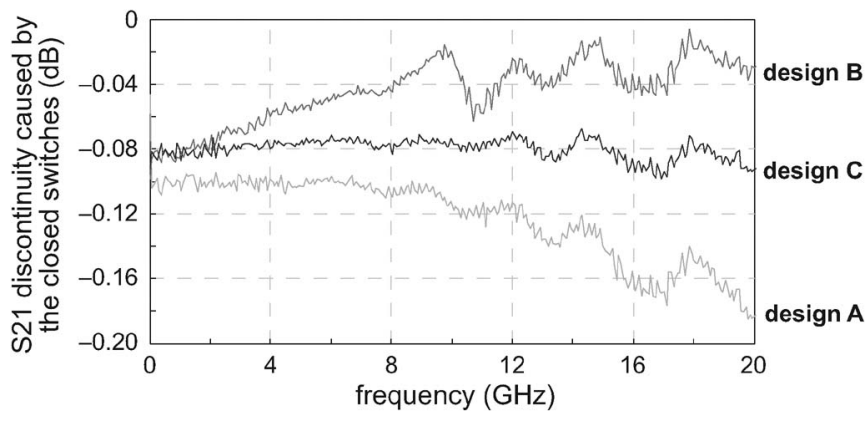

Fig. 14. Insertion loss of the three SPST switch mechanisms only, i.e., corrected by subtracting the insertion loss of their corresponding straight transmission lines.

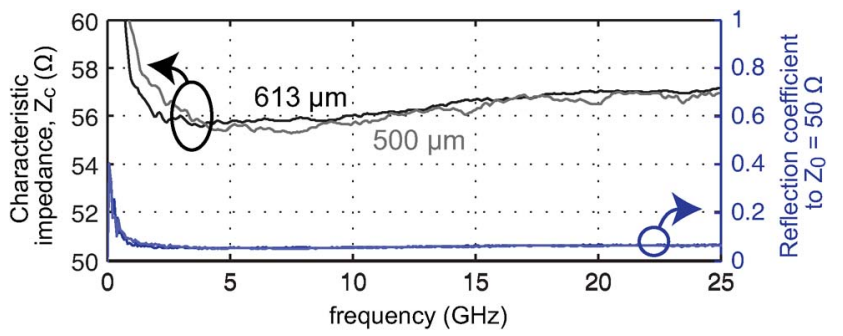

Fig. 15. Characteristic impedance of two transmission line segments of different length extracted from the measured S-parameters and line mismatched to a system impedance $Z_{0}$ of $50 \Omega$.

The loss in the 3-D micromachined transmission line alone is higher than comparable micromachined transmission lines [16] which can be explained by the inaccurate control of the gold thickness on the sidewalls and potential charging of the SOI wafer layers, which has not been further investigated. The transmission line losses could be one reason why the total insertion loss of the fabricated switches is slightly worse than that of similar switch concepts [9].

The measured characteristic impedance of the nominal $50 \Omega$ line is between $55 \Omega$ and $57 \Omega$, as extracted from the measured S-parameters of two transmission lines of different length using the formula $Z_{\mathrm{c}}= \pm Z_{0} \sqrt{\left[\left(\left(1+S_{11}\right)^{2}-S_{21}^{2}\right) /\left(\left(1-S_{11}\right)^{2}-S_{21}^{1}\right)\right]}$ [38], and plotted in Fig. 15 along with the line reflections to a nominal $50 \Omega$ system impedance.

The dc actuation voltages were measured for the different cantilever constellations to be between 23 and $39 \mathrm{~V}$, and the total dc resistance of the closed switches including their transmission lines is between 0.9 and $1.2 \Omega$ with sputtered gold contacts. The actuation electrodes of the prototype designs were electrically contacted by probe needles for opening and closing the switches. The mechanical multistability facilitates removing of the dc probe needles during RF measurements to avoid interference.

The mechanical robustness of the laterally actuated switch cantilevers has been verified, at a switching frequency of $3 \mathrm{kHz}$ with a measurement signal of $1.5 \mu \mathrm{A}$, up to 150 million hot-switching cycles, after which the tests were discontinued without observing stiction. The tests were carried out with unpackaged devices and in uncontrolled atmosphere. Since the full procedure for opening and closing of the interlocking mechanism, requiring multiple synchronized control lines, had to be performed manually for the evaluation of the prototype devices, no significant data of large cycle numbers on the reliability of the full sequence is available.

\section{B. Performance of the T-Junction Switches}

The insertion loss, isolation, and reflections of the two different design variants $\mathrm{D}$ and $\mathrm{E}$ for both the open and the closed state are plotted in Fig. 16 for different two-port measurement configurations between the three ports of the devices. For all measurements the third port was left open (unterminated). Additionally, the figure shows multiport measurements of a solid reference line.

The isolation of the open switch measured between the input (port 1) and the output (port 2) was determined to 43 and $22 \mathrm{~dB}$ at 1 and $10 \mathrm{GHz}$, respectively. The total insertion loss of the closed switch including the transition line pieces and the T-junction discontinuity is 0.31 and $0.68 \mathrm{~dB}$ at 1 and $10 \mathrm{GHz}$, respectively, and the line reflections are 29 and $22 \mathrm{~dB}$ at these frequencies. The measurements show that design $\mathrm{E}$ is more suitable for applications requiring high offstate isolation and that design $\mathrm{D}$ is more appropriate for applications requiring low insertion loss. The isolation between port 2 and 3 in the closed switch configuration (port 1 to 2) was better than $40 \mathrm{~dB}$ and $24 \mathrm{~dB}$ at $1 \mathrm{GHz}$ and $10 \mathrm{GHz}$, respectively. In the open configuration, the isolation between port 2 and 3 was better than $37 \mathrm{~dB}$ at $10 \mathrm{GHz}$. Reference measurements show that the insertion loss of a solid line T-junction (see Fig. 16) amounts to $0.15 \mathrm{~dB}$ at $1 \mathrm{GHz}$ and $0.43 \mathrm{~dB}$ at $10 \mathrm{GHz}$, and the reflections of this reference T-junction are 36 and $24 \mathrm{~dB}$ at these frequencies, respectively. This indicates that the T-junction reflections/losses are dominating over the losses attributed to the actual switch mechanism. Thus, a better RF design compensating for the geometry of the T-junction itself could further emphasize the advantages of the low-intrusive design of the signal line embedded SPDT MEMS switches.

The switch geometry has also been simulated in HFSS to determine the current distribution inside the signal lines of the two different designs. In Fig. 17(a)-(c), the magnitude of the volume current is shown at a cross-section through design $\mathrm{D}$, design E, and the solid T-junction reference line, respectively. As expected, most of the current is concentrated at the edges of the lines. In Fig. 17(d) and (e), the degree of symmetry of the current distribution is shown by plotting the integrated volume current in the left/right and top/bottom halves of the signal lines. The symmetric switch design (D) has a better balanced current distribution on the signal line of port 2, since the current is divided between the two cantilevers.

The dc actuation voltages of the T-junction switches were measured to be between 39 and $89 \mathrm{~V}$ for different cantilever lengths.

\section{CONCLUSION}

A novel MEMS switch concept has been demonstrated featuring a mechanically multistable switch mechanism with static zero-power-consumption robust active opening mechanism allowing the use of soft metal for the switch contacts, and 

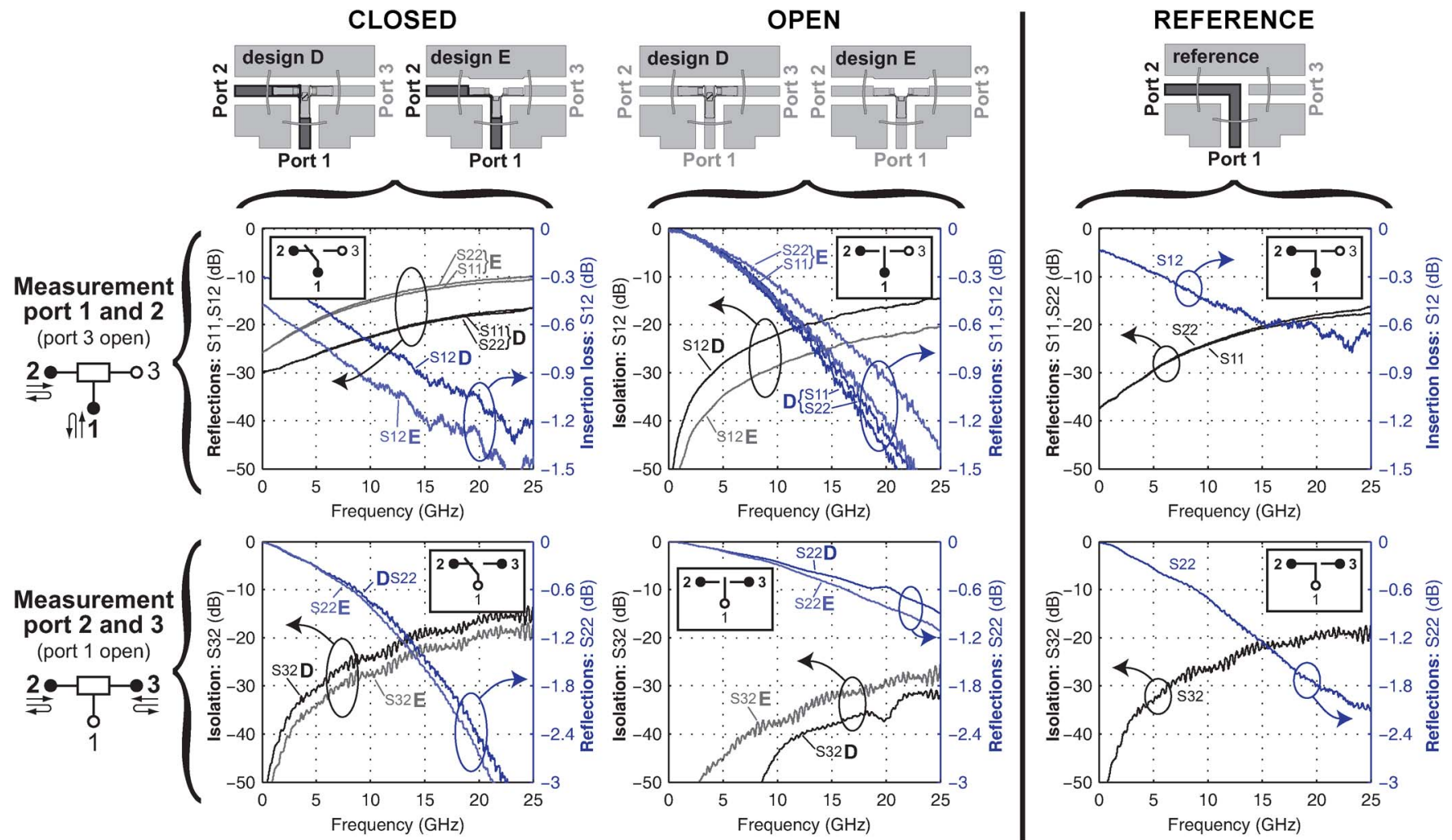

Fig. 16. Measured RF performance of the fabricated T-junction embedded SPDT switches in the open and closed state, compared to a solid signal line through the T-junction as a reference measurement. All measurements are two-port measurements with the third port left open.

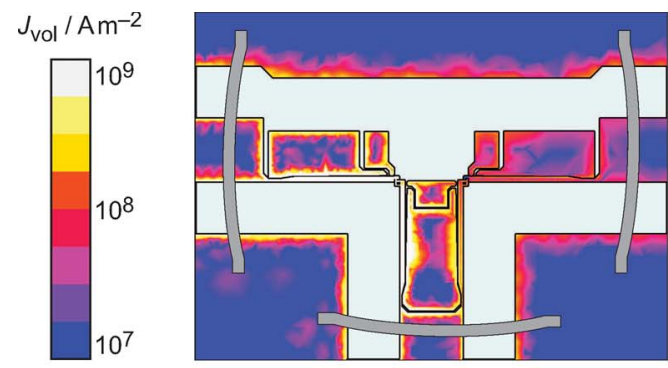

(a)

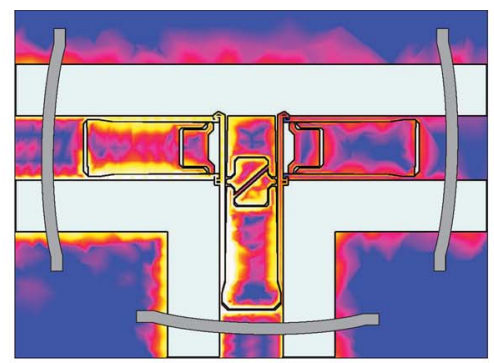

(b)

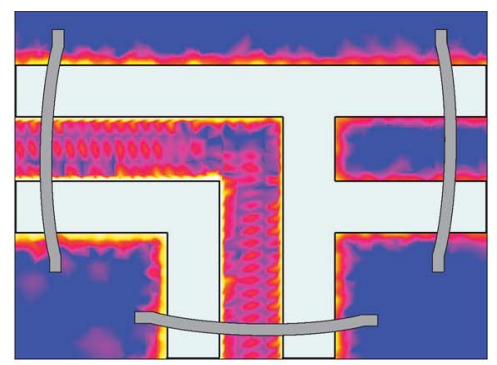

(c)

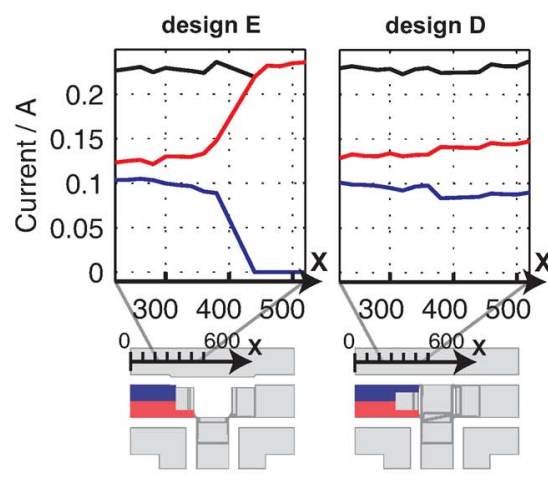

(d)
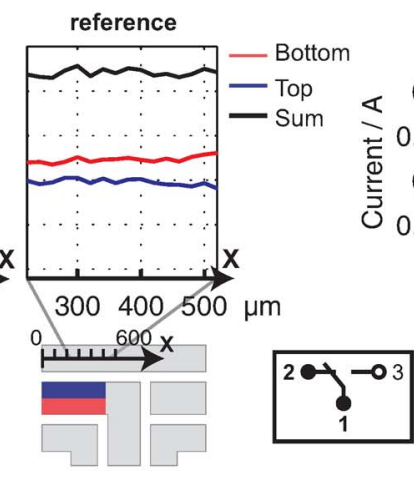

- Bottom - Top

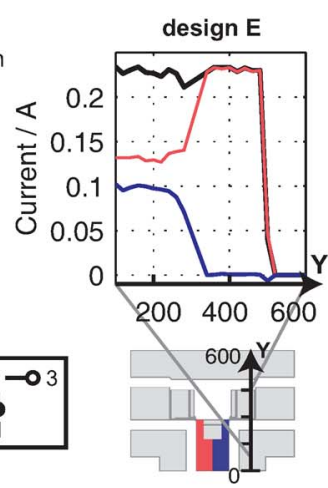

Fig. 17. Ansoft HFSS simulation of the volume current distribution inside the T-junction signal lines, shown for the two switch designs, D and E, and the solid reference line. All cases show the switch configuration closed between port 1 and port 2, and port 3 open.

low-loss RF design with the switch actuators embedded inside the signal line of a 3-D micromachined coplanar waveguide. Prototype switches in two-port and three-port configuration have been fabricated in a single photolithography process using deep reactive ion-etching, and the RF and dc performance of the switch designs have been successfully evaluated. 


\section{ACKNOWLEDGMENT}

The authors would like to thank G. Malm, IMIT, KTH, Royal Institute of Technology, Stockholm, Sweden, who kindly assisted with the RF measurements.

\section{REFERENCES}

[1] G. M. Rebeiz, RF MEMS Theory, Design and Technology, 1st ed. Hoboken, NJ: Wiley, 2003.

[2] E. R. Brown, "RF-MEMS switches for reconfigurable integrated circuits," IEEE Trans. Microw. Theory Tech., vol. 46, no. 11, pp. 1868-1880, Nov. 1998, Invited Paper.

[3] J. Yao, "RF MEMS from a device perspective," J. Micromech. Microeng., vol. 10, no. 4, pp. R9-R38, Dec. 2000.

[4] G. M. Rebeiz and J. B. Muldavin, "RF MEMS switches and switch circuits," IEEE Microw. Mag., vol. 2, no. 4, pp. 59-71, Dec. 2001.

[5] D. Hyman, J. Lam, B. Warneke, A. Schmitz, T. Y. Hsu, J. Brown, J. Schaffner, A. Walston, R. Y. Loo, M. Mehregany, and J. Lee, "Surfacemicromachined RF MEMS switches on GaAs substrates," Int. J. RF Microw. Comput.-Aided Eng., vol. 9, no. 4, pp. 348-361, Jul. 1999.

[6] S. Shen and M. Feng, "Low actuation voltage RF MEMS switches with signal frequencies from $0.25 \mathrm{GHz}$ to $40 \mathrm{GHz}$," in IEDM Tech. Dig., Washington, DC, Dec. 1999, pp. 689-692.

[7] J. Muldavin and G. Rebeiz, "Inline capacitive and DC-contact MEMS shunt switches," IEEE Microw. Wireless Compon. Lett., vol. 11, no. 8, pp. 334-336, Aug. 2001.

[8] I. Schiele and B. Hillerich, "Comparison of lateral and vertical switches for application as microrelays," J. Micromech. Microeng., vol. 9, no. 2, pp. 146-150, Jun. 1999.

[9] A. Liu, M. Tang, A. Agarwal, and A. Alphones, "Low-loss lateral micromachined switches for high frequency applications," J. Micromech. Microeng., vol. 15, no. 1, pp. 157-167, Jan. 2005.

[10] J. Oberhammer and G. Stemme, "Active opening force and passive contact force electrostatic switches for soft metal contact materials," J. Microelectromech. Syst., vol. 15, no. 5, pp. 1235-1242, Oct. 2006.

[11] V. Milanovic, M. Maharbiz, A. Singh, B. Warneke, N. Zhou, H. K. Chan, and K. S. J. Pister, "Microrelays for batch transfer integration in RF systems," in Proc. IEEE Int. Conf. MEMS, Miyazaki, Japan, Jan. 2000, pp. 787-792.

[12] D. Hah, E. Yoon, and S. Hong, "A low voltage actuated micromachined microwave switch using torsion springs and leverage," in Proc. IEEE MTT-S Int. Microw. Symp. Dig., Boston, MA, Jun. 2000, vol. 1, pp. $157-160$.

[13] M. Sterner, N. Roxhed, G. Stemme, and J. Oberhammer, "Mechanically bi-stable in-plane switch with dual-stiffness actuators," in Proc. Int. SolidState Sens., Actuators, Microsyst. Conf. (TRANSDUCERS), Lyon, France, Jun. 2007, vol. 2, pp. 1401-1404.

[14] J. Oberhammer, M. Tang, A.-Q. Liu, and G. Stemme, "Mechanically tristable, true single-pole-double-throw (SPDT) switches," J. Micromech. Microeng., vol. 16, no. 11, pp. 2251-2258, Nov. 2006.

[15] P. Blondy, A. Brown, D. Cros, and G. Rebeiz, "Low-loss micromachined filters for millimeter-wave communication systems," IEEE Trans. Microw. Theory Tech., vol. 46, no. 12, pp. 2283-2288, Dec. 1998.

[16] K. Herrick, T. Schwarz, and L. Katehi, "Si-micromachined coplanar waveguides for use in high-frequency circuits," IEEE Trans. Microw. Theory Tech., vol. 46, no. 6, pp. 762-768, Jun. 1998.

[17] V. Milanovic, M. Gaitan, E. Bowen, and M. Zaghloul, "Micromachined microwave transmission lines in CMOS technology," IEEE Trans. Microw. Theory Tech., vol. 45, no. 5, pp. 630-635, May 1997.

[18] B. Shenouda, L. Pearson, and J. Harriss, "Etched-silicon micromachined W-band waveguides and horn antennas," IEEE Trans. Microw. Theory Tech., vol. 49, no. 4, pp. 724-727, Apr. 2001.

[19] K. Takahashi, U. Sangawa, S. Fujita, M. Matsuo, T. Urabe, H. Ogura, and H. Yabuki, "Packaging using microelectromechanical technologies and planar components," IEEE Trans. Microw. Theory Tech., vol. 49, no. 11, pp. 2099-2104, Nov. 2001.

[20] Y. Kwon, H.-T. Kim, J.-H. Park, and Y.-K. Kim, "Low-loss micromachined inverted overlay CPW lines with wide impedance ranges and inherent airbridge connection capability," IEEE Microw. Wireless Compon. Lett., vol. 11, no. 2, pp. 59-61, Feb. 2001.

[21] H.-S. Lee, S.-C. Kim, B.-O. Lim, K.-M. Kim, W.-Y. Uhm, Y.-H. Chun, D.-H. Shin, S.-K. Kim, H.-C. Park, and J.-K. Rhee, "New micromachined microstrip transmission lines for application in millimeter-wave circuits," Microw. Opt. Technol. Lett., vol. 40, no. 1, pp. 6-9, Jan. 2004.
[22] H.-T. Kim, S. Jung, J.-H. Park, C.-W. Baek, Y.-K. Kim, and Y. Kwon, "A new micromachined overlay CPW structure with low attenuation over wide impedance ranges and its application to low-pass filters," IEEE Trans. Microw. Theory Tech., vol. 49, no. 9, pp. 1634-1639, Sep. 2001.

[23] Y. Kim, I. Llamas-Garro, C.-W. Baek, and Y.-K. Kim, "A monolithic surface micromachined half-coaxial transmission line filter," in Proc. IEEE Int. Conf. MEMS, Istanbul, Turkey, Jan. 2006, pp. 870-873.

[24] K. Jiang, M. Lancaster, I. Llamas-Garro, and P. Jin, "SU-8 Ka-band filter and its microfabrication," J. Micromech. Microeng., vol. 15, no. 8, pp. 1522-1526, Aug. 2005.

[25] R. Chen, E. Brown, and C. Bang, "A compact low-loss Ka-band filter using 3-dimensional micromachined integrated coax," in Proc. IEEE Int. Conf. MEMS, Maastricht, The Netherlands, Jan. 2004, pp. 801-804.

[26] D. Newlin, A.-V. Pham, and J. Harriss, "Development of low loss organic-micromachined interconnects on silicon at microwave frequencies," IEEE Trans. Compon. Packag. Technol., vol. 25, no. 3, pp. 506-510, Sep. 2002.

[27] J. Bishop, M. Hashemi, K. Kiziloglu, L. Larson, N. Dagli, and U. Mishra, "Monolithic coaxial transmission lines for mm-wave ICs," in Proc. IEEE/Cornell Conf. Adv. Concepts High Speed Semicond. Devices Circuits, Ithaca, NY, Aug. 1991, pp. 252-260.

[28] M. A. Forman, "Low-loss LIGA-fabricated coplanar waveguide and filter," in Proc. APMC, Yokohama, Japan, Dec. 2006, pp. 1905-1907.

[29] M. Scardelletti, G. Ponchak, and N. Varaljay, "MEMS, Ka-band singlepole double-throw (SPDT) switch for switched line phase shifters," in Proc. IEEE Antennas Propag. Soc. Int. Symp., San Antonio, TX, Jun. 2002, vol. 2, pp. 2-5.

[30] B. Schauwecker, K. Strohm, T. Mack, W. Simon, and J.-F. Luy, "Singlepole-double-throw switch based on toggle switch," Electron. Lett., vol. 39, no. 8, pp. 668-670, Apr. 2003.

[31] M. Tang, A.-Q. Liu, A. Agarwal, Z.-S. Liu, and C. Lu, "A singlepole double-throw (SPDT) circuit using lateral metal-contact micromachined switches," Sens. Actuators A, Phys., vol. 121, no. 1, pp. 187-196, Feb. 2005.

[32] I.-J. Cho, T. Song, S.-H. Baek, and E. Yoon, "A low-voltage push-pull SPDT RF MEMS switch operated by combination of electromagnetic actuation and electrostatic hold," in Proc. IEEE Int. Conf. MEMS, Miami, FL, Jan. 2005, pp. 32-35.

[33] M. Sterner, N. Roxhed, G. Stemme, and J. Oberhammer, "Coplanarwaveguide embedded mechanically-bistable DC-to-RF MEMS switches," in Proc. IEEE MTT-S Int. Microw. Symp. Dig., Honolulu, HI, Jun. 2007, pp. 359-362.

[34] M. Sterner, N. Roxhed, G. Stemme, and J. Oberhammer, "Mechanically tri-stable SPDT metal-contact MEMS switch embedded in 3D transmission line," in Proc. IEEE EuMC, Munich, Germany, Oct. 2007, pp. $1225-1228$.

[35] R. A. Coutu, Jr., P. E. Kladitis, K. D. Leedy, and R. L. Crane, "Selecting metal alloy electric contact materials for MEMS switches," J. Micromech. Microeng., vol. 14, no. 8, pp. 1157-1164, Aug. 2004.

[36] R. Collin, Foundation for Microwave Engineering. New York: McGrawHill, 1966.

[37] M. Sterner, N. Roxhed, G. Stemme, and J. Oberhammer, "Maskless selective electrochemically assisted wet etching of metal layers for 3D micromachined SOI RF MEMS devices," in Proc. IEEE Int. Conf. MEMS, Tucson, AZ, Jan. 2008, pp. 383-386.

[38] J. Zhang and T. Y. Hsiang, "Extraction of subterahertz transmissionline parameters of coplanar waveguides," PIERS Online, vol. 3, no. 7, pp. 1102-1106, 2007.

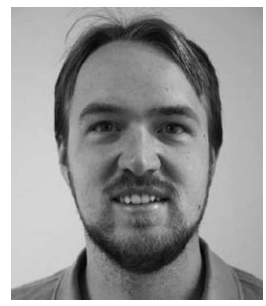

Mikael Sterner was born in Stockholm, Sweden, in 1981. He received the M.Sc. degree in engineering physics from Royal Institute of Technology (KTH), Stockholm, in 2006. He has been working toward the Ph.D. degree in microsystems technology at the Microsystem Technology Lab, KTH, since 2006.

His main research fields are RF MEMS switches and microwave MEMS tuneable high-impedance surfaces. 


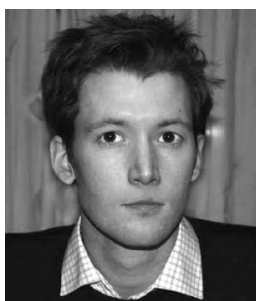

Niclas Roxhed (M'09) was born in Stockholm, Sweden, in 1978. He received the M.Sc. degree in electrical engineering and the Ph.D. degree in microsystem technology from the KTH, Stockholm, in 2003 and 2007, respectively.

He is currently a Research Associate leading research activities medical-related MEMS. He has authored or coauthored 28 scientific papers. His main research fields are sensors for medical diagnostics and medical-aid microsystems. He is also involved in high precision etching using DRIE for RF-MEMS switches and 3-D integration of MEMS on ICs for infrared imagers.

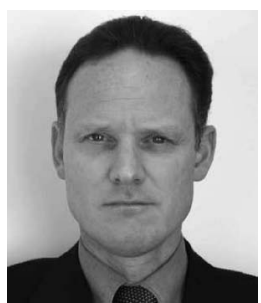

Göran Stemme (M'98-SM'04-F'06) received the M.Sc. degree in electrical engineering and the Ph.D. degree in solid-state electronics from the Chalmers University of Technology, Gothenburg, Sweden, in 1981 and 1987, respectively.

In 1981, he joined the Department of Solid State Electronics, Chalmers University of Technology. There, in 1990, he became an Associate Professor (docent) heading the silicon sensor research group. In 1991, he was appointed Professor at the KTH, Stockholm, Sweden, where he heads the Microsystem Technology group at the School of Electrical Engineering. He has published more than 260 research journal and conference papers and has more than 22 patents proposals or granted patents. His research is devoted to microsystem technology based on micromachining of silicon. The works spans over a broad range of technological and application fields such as medical technology, biochemistry, biotechnology, microfluidics, optical applications, wafer-level packaging, and device integration. Some of the results have successfully been commercialized.

Dr. Stemme is a member of the Royal Swedish Academy of Sciences (KVA). He is a member of the Editorial Board of the IEEE/ASME JOURNAL of Microelectromechnical Systems since 1997 and was a member of the the Editorial Board of the Royal Society of Chemistry journal "Lab On A Chip" between 2000 and 2005. Between 1995 and 2001, he was a member of the International Steering Committee of the Conference series IEEE Microelectromechanical Systems and he was General Cochair of that conference in 1998. In 2001, he won, together with two colleagues, the final of Innovation Cup in Sweden.

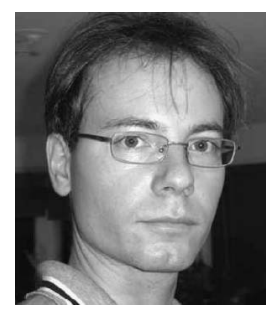

Joachim Oberhammer (M'06) was born in Italy, in 1976. He received the M.Sc. degree in electrical engineering from the Graz University of Technology, Graz, Austria, in 2000. He received the Ph.D. degree for his work on RF MEMS switches and microsystem packaging from KTH, Stockholm, Sweden, in 2004.

He was working with automotive sensor electronics and RFID systems with the Graz University of Technology and Vienna University of Technology, Vienna, Austria, before he joined the Microsystem Technology Laboratory, KTH. After having been a Post-Doctoral Research Fellow at Nanyang Technological University, Singapore, he returned to the KTH in 2005. In 2007, he became an Associate Professor at the KTH, where he is heading a research team with activities in RF and microwave MEMS. In 2007 and 2008, respectively, he was a Guest Researcher at Nanyang Technological University, and at Kyoto University, Kyoto, Japan. He is author or a coauthor of more than 50 reviewed research papers and holds four patents.

Dr. Oberhammer got the award of the Ericsson Research Foundation in 2004, and received a scholarship by the Japanese Society for the Promotion of Science in 2007, as well as the best paper award at IEEE European Microwave Integrated Circuits Conference 2009. 